\title{
Efficient Particle Separation in Microfluid Channel with Inhomogeneous Magnetic Field
}

\author{
Brian Nlong Zhao \\ University of Southern California, Los Angeles, CA, USA \\ Email: briannlongzhao@gmail.com
}

How to cite this paper: Zhao, B.N. (2019) Efficient Particle Separation in Microfluid Channel with Inhomogeneous Magnetic Field. Journal of Electromagnetic Analysis and Applications, 11, 17-23.

https://doi.org/10.4236/jemaa.2019.112002

Received: January 29, 2019

Accepted: February 25, 2019

Published: February 28, 2019

Copyright $\odot 2019$ by author(s) and Scientific Research Publishing Inc. This work is licensed under the Creative Commons Attribution International License (CC BY 4.0).

http://creativecommons.org/licenses/by/4.0/

\begin{abstract}
In this paper, we combined magnetostatics and laminar flow in microfluidics and studied a particle separation scheme employing magnetophoretic force in inhomogeneous magnetic field. A detailed model and analysis is shown and the proposed scheme is capable of efficiently separating magnetic particles with different permeability and sizes. The method shows a way to separate efficient particles and could potentially be implemented in biological and chemical systems.
\end{abstract}

\section{Keywords}

Particle Separation, Efficient Separation, Microfluid Channel, Inhomogeneous Magnetic Field

\section{Introduction}

Separating different kinds of chemicals or biological particles from a mixture is often an important and significant step in conducting research and manipulating a specific type of particle. By harnessing the unique properties of microfluidics, techniques have been developed for fast and efficient separation and sorting in diagnostics, chemical and biological analyses, food and chemical processing, and environmental assessment [1] [2] [3] [4] [5]. Various methods using different mechanism have evolved, including dielectrophoresis, inertial microfluidics, acoustics, and optics [6] [7] [8] [9] [10]. Conversely, using magnetophoretic force to control the movement of particles in fluid is a convenient technique to separate micro- and nano particles in fluid under an inhomogeneous magnetic field. Magnetophoretic force is exerted on a magnetic particle induced by the gradient of an inhomogeneous magnetic field. Through this method, particles can be easily separated without physical contact with fluid and particles. 
In this paper, we will introduce the basics of applying magnetophoretic force for particle separation and propose a model to simulate the separation of two particles with different properties in a microfluid channel using this method.

\section{Theoretical Background}

Our model requires two different physics interfaces with the first one being magnetostatics. The Gauss's law for magnetism is the following.

$$
\nabla \cdot B=0
$$

This law states that the total magnetic flux through a closed surface is zero. This indicates that the magnetic field has no source, and field lines must be closed. In other words, it implies that the magnetic monopole does not exist. As a result, another equation is necessary to solve the magnetic field. Maxwell-Ampère's law without electric field states the relationship between the magnetic field $(H)$ and current density $(\lambda)[11]$ :

$$
\nabla \times H=J
$$

The magnetophoretic force is the force that imposes permeable particles toward regions where the magnetic field is inhomogeneous. This force is applicable for particles which are neutral and with a relative permeability are different from the background fluid. The magnetophoretic force is expressed as the following equation [12]:

$$
F_{m}=2 \pi r_{p}^{3} \mu_{0} \mu_{r, f} K \nabla|H|^{2}
$$

where $H$ is the magnetic field, $\mu_{t, f}$ is the relative permeability of the fluid, $\mu_{t, p}$ is the relative permeability of the particle, and $K$ is the Clausius-Mossotti factor, defined as:

$$
K=\frac{\mu_{r, p}-\mu_{r, f}}{\mu_{r, p}+2 \mu_{r, f}}
$$

The Stokes drag force, which is proportional to the relative velocity between the particles and the fluid is expressed as the following [13]:

$$
F_{\text {drag }}=\frac{18 \mu}{\rho_{p} d_{p}^{2}} m_{p}\left(u_{f}-v_{p}\right)
$$

where $m_{p}$ is the particle mass in unit of $\mathrm{kg} . \mu$ is the fluid viscosity with unit of Pa.s. $\rho_{p}$ is the particle density with unit of $\mathrm{kg} / \mathrm{m}^{3} . d_{p}$ is the particle diameter with unit of $\mathrm{m} . \boldsymbol{v}_{p}$ is the velocity of the particle in unit of $\mathrm{m} / \mathrm{s}$, and $\boldsymbol{u}_{f}$ is the fluid velocity in unit of $\mathrm{m} / \mathrm{s}$.

\section{Model Establishment}

\subsection{The Geometry of the Model}

The model is two dimensional instead of three dimensional, since it is easier to simulate and can show the mechanism behind it. We first build the model by creating the background of the simulation and the Y-shaped fluid channel used for separating particles in the fluid into two streams. The background is a $2000 \times$ 
$4000 \mu \mathrm{m}$ rectangle and is filled with air. Next, a Y-shape is in the background we previously built. The width of the path for this fluid channel is $200 \mu \mathrm{m}$ throughout the entire path. The length of the main path is $2000 \mu \mathrm{m}$, and the length of the branches is $500 \mu \mathrm{m}$. This shape would be the fluid channel that allows fluid to flow inside from the left and exit through two ends at the right. Then, we draw eight rectangular shapes above the Y-shape and keep a small distance between the rectangles and the Y-shaped fluid channel. These boxes serve as magnets to generate the magnetic field in our model. Each magnet has the size of 500 $\times 100 \mu \mathrm{m}$, separated by $100 \mu \mathrm{m}$ from each other. The corners of the magnets and the fluid channel are also smooth, so the computation complexity of the force and field would be reduced. A sketch of the geometry of our model is shown below in Figure 1.

\subsection{The Magnetic Field}

We first set the background of the magnetic property. The boundary of the background is set to be magnetic insulative, so the magnetic field can only be calculated inside the domain bounded by the boundary of the background. The initial value of the magnetic field within the domain is set to zero, which means that there is no magnetic field at the initial state other than the field that will be generated by the magnets. The permeability of the background is set to $\mu_{0}$, which is the permeability of free space. For the magnets, the remanent flux density is set to $1 \mathrm{~T}$ in $\mathrm{y}$-direction, so the direction of magnetization of particles could result in a force difference in the vertical direction, which therefore separates the particles vertically. The relative permittivity of our domain is set to 1 . Since no electric filed will be involved in our simulation, the value of permittivity will not alter the result of the simulation.

\subsection{Microfluid in Laminar Flow}

First, we set the global properties and reference values of the fluid. The fluid is set to be an incompressible laminar flow, which means no turbulence will be involved, and the density of fluid remains constant. The pressure and temperature are set under normal room condition, which are $1 \mathrm{~atm}$ and $293.15 \mathrm{~K}$, respectively. In this model, we use water as the fluid, which applies density and dynamic viscosity of water into our simulation.

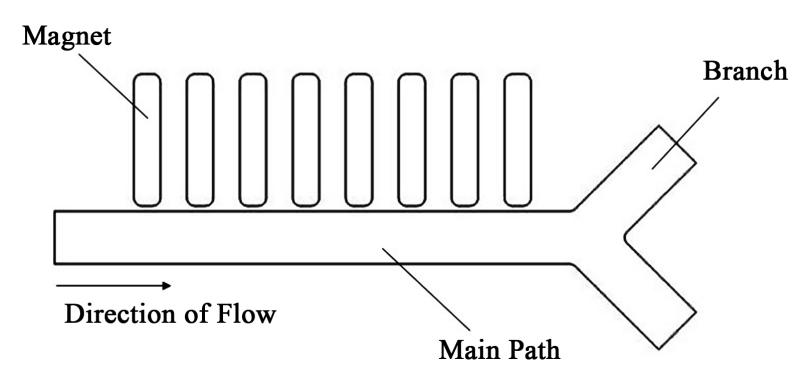

Figure 1. The geometry of the model under investigation. The fluid and particles are injected from the left inlet. 
The left end of the fluid channel is defined as inlet of water, and initial velocity is set to $250 \mu \mathrm{m} / \mathrm{s}$ to the right. Water will flow through the fluid channel from inlet to two outlets at the right end of the Y-shaped fluid channel. At the wall of the fluid channel, the velocity of the fluid is set to zero.

\subsection{Particle Tracing in Fluid Flow}

We place two different kinds of particles with different sizes and relative permeability at the inlet of the fluid channel. Particle 1 has a diameter of $15 \mu \mathrm{m}$ and a permeability slightly higher than the permeability of free space, while particle 2 has a diameter of $10 \mu \mathrm{m}$ and a permeability lower than the permeability of free space. Both of them have a density of $1050 \mathrm{~kg} / \mathrm{m}^{3}$, however, due to the relatively small mass, the effect of gravity on these two particles cannot be ignored. The drag force on the particles is applied based on Stokes' law through the introduction of properties of the fluid, so the particles will move along with the flow of the fluid flow and exit through the outlets. Then, the magnetophoretic force on the particles could be calculated by applying the magnetic field, the fluid properties, and the particle properties. Thus, we could conduct the simulation and generate trajectories of the two different types of particles.

\section{Results and Discussions}

Figure 2 shows the magnetic field that results from the remanent flux density of the magnets. It also shows that all the magnetic field lines are closed curves, which is in line with Gauss's law for magnetism.

Figure 3 and Figure 4 show the velocity and pressure of fluid inside the fluid channel, respectively. According to the defined boundary, the velocity of the fluid is zero at the wall of the fluid channel, which could be seen from the blue color near the wall of the fluid channel. The velocity at the center of the channel reaches its maximum value of $350 \mathrm{um} / \mathrm{s}$. The pressure goes down almost linear along the fluid flow and is zero at the two outlets where no external pressure is imposed.

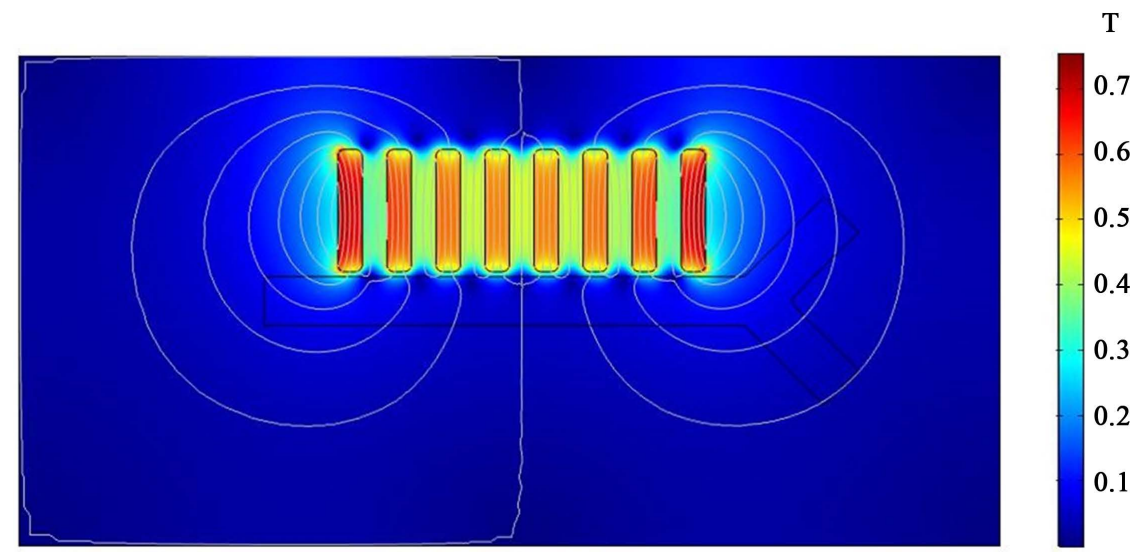

Figure 2. The magnetic field strength near the magnets and the contour plot of the magnetic field lines generated by the magnets. 
Finally, particle trajectories (denoted as red and blue) under the influence of magnetophoretic force are shown in Figure 5. These particles are assumed to be released at the center of the inlet. As they propagate along the channel, the magnetophoretic force applied in the y-direction alters their path according to their relative permeability. After the impact of magnetophoretic force, the two particles go into different outlets as desired.

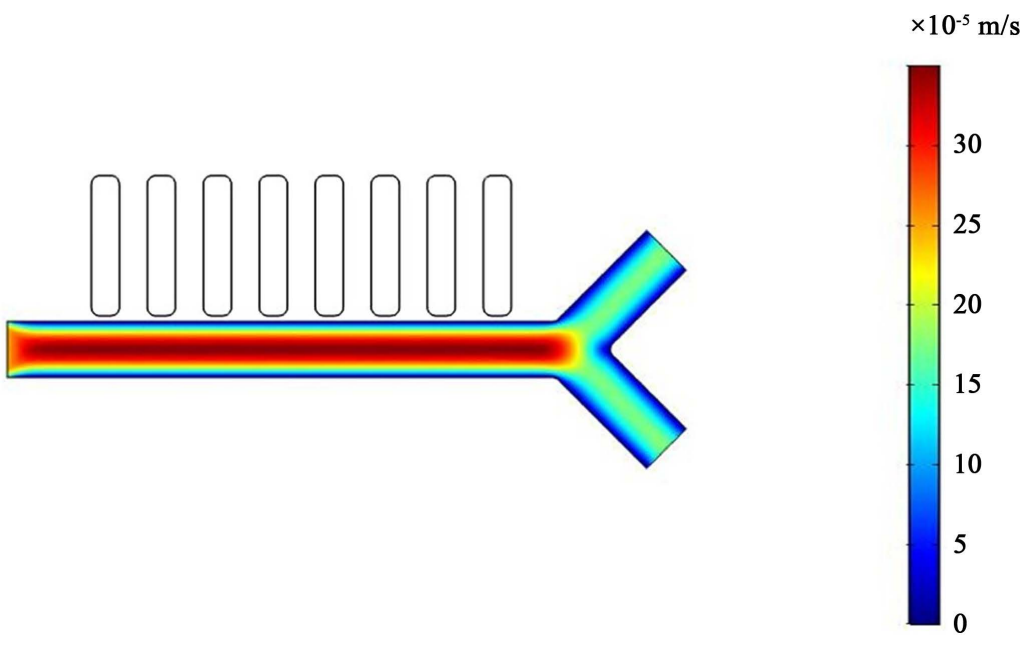

Figure 3. The velocity of fluid inside the fluid channel.

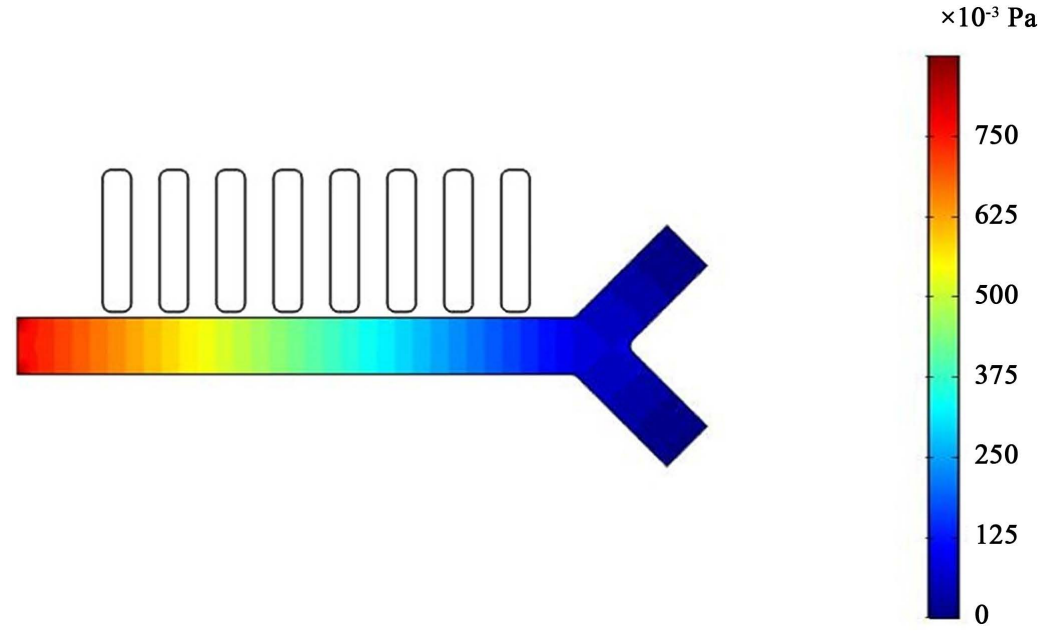

Figure 4. The pressure distribution of fluid inside the fluid channel.

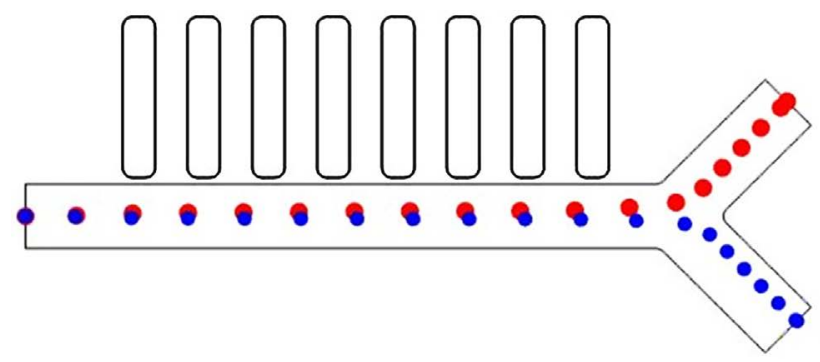

- Particle A

Figure 5. The trajectories of two particles in the fluid. 


\section{Conclusion}

In conclusion, we have proposed an efficient scheme for particle separation in microfluidics under the influence of the inhomogeneous magnetic field. The method discussed different field distribution and particle trajectories in detail. Further study could be performed on different particle and fluid channel designs for future lab-on-a-chip devices.

\section{Conflicts of Interest}

The author declares no conflicts of interest regarding the publication of this paper.

\section{References}

[1] Huh, D., Bahng, J.H., Ling, Y., Wei, H.-H., Kripfgans, O.D., Fowlkes, J.B., Grotberg, J.B. and Takayama, S. (2007) Gravity-Driven Microfluidic Particle Sorting Device with Hydrodynamic Separation Amplification. Analytical Chemistry, 79, 1369-1376. https://doi.org/10.1021/ac061542n

[2] Petersson, F., Åberg, L., Swärd-Nilsson, A.-M. and Laurell, T. (2007) Free Flow Acoustophoresis: Microfluidic-Based Mode of Particle and Cell Separation. Analytical Chemistry, 79, 5117-5123. https://doi.org/10.1021/ac070444e

[3] Gossett, D.R., Weaver, W.M., Mach, A.J., Hur, S.C., Tse, H.T.K., Lee, W., Amini, H. and Di Carlo, D. (2010) Label-Free Cell Separation and Sorting in Microfluidic Systems. Analytical and Bioanalytical Chemistry, 397, 3249-3267. https://doi.org/10.1007/s00216-010-3721-9

[4] Bhagat, A.A.S., Bow, H., Hou, H.W., Tan, S.J., Han, J. and Lim, C.T. (2010) Microfluidics for Cell Separation. Medical \& Biological Engineering \& Computing, 48, 999-1014. https://doi.org/10.1007/s11517-010-0611-4

[5] Xia, N., Hunt, T.P., Mayers, B.T., Alsberg, E., Whitesides, G.M., Westervelt, R.M. and Ingber, D.E. (2006) Combined Microfluidic-Micromagnetic Separation of Living Cells in Continuous Flow. Biomedical Microdevices, 8, 299. https://doi.org/10.1007/s10544-006-0033-0

[6] Gascoyne, P.R.C. and Vykoukal, J. (2002) Particle Separation by Dielectrophoresis. Electrophoresis, 23, 1973-1983. https://doi.org/10.1002/1522-2683(200207)23:13<1973::AID-ELPS1973>3.0.CO;2-1

[7] Kuntaegowdanahalli, S.S., Bhagat, A.A.S., Kumar, G. and Papautsky, I. (2009) Inertial Microfluidics for Continuous Particle Separation in Spiral Microchannels. Lab on a Chip, 9, 2973-2980. https://doi.org/10.1039/b908271a

[8] Sajeesh, P. and Sen, A.K. (2014) Particle Separation and Sorting in Microfluidic Devices: A Review. Microfluidics and Nanofluidics, 17, 1-52. https://doi.org/10.1007/s10404-013-1291-9

[9] De Sarabia, E.R.-F., Gallego-Juárez, J.A., Rodríguez-Corral, G., Elvira-Segura, L. and González-Gómez, I. (2000) Application of High-Power Ultrasound to Enhance Fluid/Solid Particle Separation Processes. Ultrasonics, 38, 642-646. https://doi.org/10.1016/S0041-624X(99)00129-8

[10] Inglis, D.W. (2009) Efficient Microfluidic Particle Separation Arrays. Applied Physics Letters, 94, 013510. https://doi.org/10.1063/1.3068750

[11] Ida, N. (2000) Engineering Electromagnetics. Vol. 2. Springer, New York. https://doi.org/10.1007/978-1-4757-3287-0 
[12] Furlani, E.P. and Sahoo, Y. (2006) Analytical Model for the Magnetic Field and Force in a Magnetophoretic Microsystem. Journal of Physics D: Applied Physics, 39, 1724. https://doi.org/10.1088/0022-3727/39/9/003

[13] Gerhart, P.M., Gerhart, A.L. and Hochstein, J.I. (2016) Munson, Young and Okiishi's Fundamentals of Fluid Mechanics, Binder Ready Version. John Wiley \& Sons, Hoboken. 